\title{
Spatial heterogeneity of piezoelectric properties in fatigued lead zirconate titanate ceramics
}

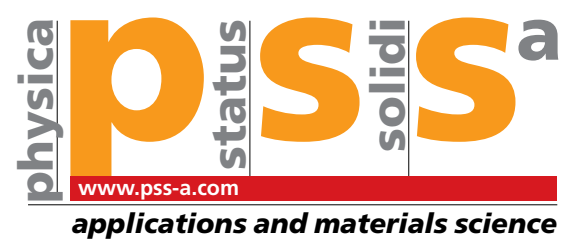

\author{
Yong Zhang ${ }^{*, 1}$, Liang Ma', Xiangrong Wang', Baohua Wen², Xiaolin Liư ${ }^{2}$, and Ivan Baturin ${ }^{3}$ \\ ${ }^{1}$ Beijing Fine Ceramics Laboratory, State Key Laboratory of New Ceramics and Fine Processing, Institute of Nuclear and New Energy \\ Technology, Tsinghua University, 100084 Beijing, P.R. China \\ ${ }^{2}$ State Key Laboratory of Organic-Inorganic Composites, Beijing University of Chemical Technology, 100029 Beijing, P.R. China \\ ${ }^{3}$ Ferroelectric Laboratory, Institute of Natural Science, Ural Federal University, 620000 Ekaterinburg, Russia
}

Received 26 December 2012, revised 26 June 2013, accepted 29 July 2013

Published online 29 August 2013

Keywords ceramics, fatigue, piezoelectric properties, switching, titanates

*Corresponding author: e-mail yzhang@tsinghua.edu.cn, Phone: +86 108019 4055, Fax: +86 1089796022

A spatial non-uniformity of the switching properties during the fatigue cycling in lead zirconate titanate ceramics was investigated by a quasi-static piezoelectric and a polarization switching measurements. The agreement between the local piezoelectric properties and the switching behavior of segmented samples was demonstrated. The observed spatial variation of the properties and its evolution with cycle number provides clear evidence of the presence of heterogeneous regions that possess a local fatigue state and the local switching behavior. These results can be explained as a result of the buildup of the spatially non-uniform field and the formation of frozen domains in the ceramics during cycling. The statistical analysis of spatial variation of the switching properties and its evolution with cycle number provides the evidence that the heterogeneity of the switching properties during the fatigue cycling in lead zirconate titanate ceramics is mostly related to the non-uniform change of the local characteristic switching time.

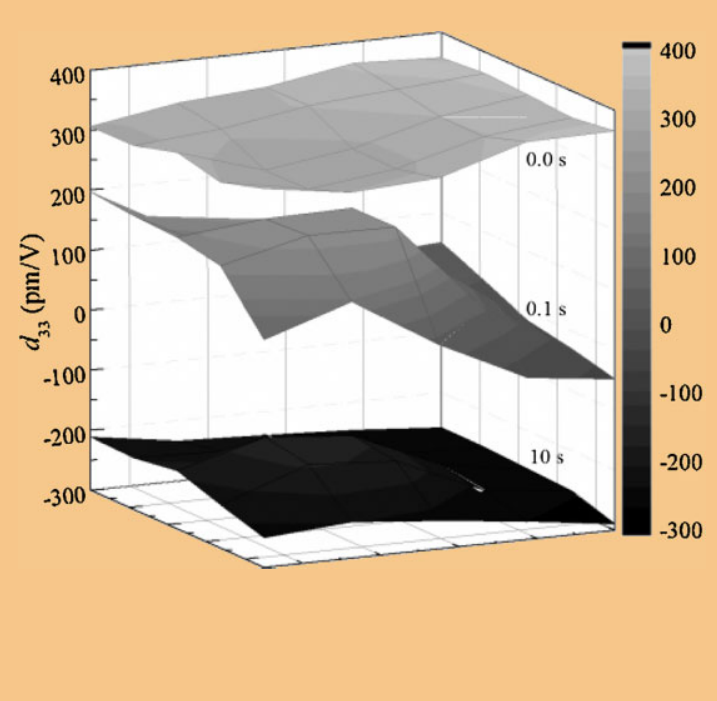

(c) 2013 WILEY-VCH Verlag GmbH \& Co. KGaA, Weinheim
1 Introduction For superior dielectric, piezoelectric, and ferroelectric properties, lead zirconate titanate (PZT) ceramics have been widely used in different fields since their discovery, especially as transducer and actuator materials. However, their successful utilization in multilayer actuator applications was originally hindered in part by the decrease of remnant polarization $P_{\mathrm{r}}$ during cyclic electrical or mechanical loading, known as fatigue effect $[1,2]$. The PZT ceramics exhibit the strong fatigue effect at high cycle numbers especially when used with metal electrodes [3, 4]. The mechanisms responsible for the reduction of the polarization during cycling, albeit extensively investigated in large number of contributions [5-7], remain unclear.
Therefore, studies on how fatigue distributes and develops in the ferroelectric ceramics are needed, in order to improve the properties and find the possibility of practical solutions for their widespread applications. There have been many earlier attempts to investigate the ferroelectric fatigue mechanism, and many conclusions are made, indicating that fatigue is caused by charge trapping and defects generated during the cyclic loading. Recently, it was shown that the progress of fatigue process is spatially heterogeneous.

Apart from application driven interests to find optimal compositions and processing parameters for the PZT ceramics, the study on fatigue heterogeneity may also help to gain the better comprehension of the polarization 
switching. A combined investigation of the dynamics of polarization switching and the fatigue heterogeneity is needed for the further study of the fatigue effect in the PZT ceramics.

Since the values of piezoelectric constant are coupled to the polarization state thus changing during polarization reversal, direct measurement of $d_{33}$ values using a quasistatic method $[8,9]$ can provide a useful clue for general understanding of polarization switching phenomena and switching retardation due to the fatigue in the PZT ceramics. Moreover, the piezoelectric properties can be measured locally providing information about a spatial distribution of the polarization including switchable and non-switchable parts. It was shown that the properties vary substantially with the spatial position in the sample during the fatigue cycling $[8,10]$. Although the previous works [8-10] were focused on the study of heterogeneity of the small-signal and large-signal properties in fatigued samples, limited attention was paid to statistical analysis of such heterogeneity. Moreover, most of the results were obtained by hysteresis loop measurements without detailed study of switching kinetics in rectangular pulses which can be done by the direct measurement of the switching charge and the local $d_{33}$ values.

The present work is aimed to characterize the heterogeneity of the polarization switching process in the fatigued PZT ceramics with a focus on the spatially and time resolved $d_{33}$ and switching charge measurements accompanied by the statistical analysis of the heterogeneity.

\section{Experimental procedures}

2.1 Sample preparation It is well known that PZT ceramics have superior dielectric and piezoelectric properties when their $\mathrm{Zr} / \mathrm{Ti}$ ratio is near to a morphotropic phase boundary (MPB) at which the $\mathrm{Zr} / \mathrm{Ti}$ ratio is $52 / 48$ [11]. Furthermore, the dielectric properties of the PZT ceramics can be improved by soft-doping with $\mathrm{Nb}^{5+}$ [12]. Ceramic samples with a composition of $\mathrm{Pb}\left(\mathrm{Zr}_{0.525} \mathrm{Ti}_{0.475}\right)_{0.99} \mathrm{Nb}_{0.01} \mathrm{O}_{3}$ were chosen for the present study. The perovskite PZT ceramics were prepared using the conventional mixed-oxide method. High purity raw materials (AR, Beijing Sinopharm chemical reagent Co. Ltd., Beijing, China) of $\mathrm{PbO}, \mathrm{ZrO}_{2}$, $\mathrm{TiO}_{2}$, and $\mathrm{Nb}_{2} \mathrm{O}_{5}$ were weighed and mixed by zirconia balls in polyethylene jar for $3 \mathrm{~h}$ using a planetary mill and alcohol as a milling medium. To compensate for a $\mathrm{PbO}$ volatility during the calcination, additional $1.5 \mathrm{wt} \%$ of $\mathrm{PbO}$ was added. The slurry was dried and calcined in an alumina crucible at $800{ }^{\circ} \mathrm{C}$ for $2 \mathrm{~h}$. The calcined powder was ball-milled again for $3 \mathrm{~h}$ in the planetary mill, dried and pressed into disk pellets $20 \mathrm{~mm}$ in diameter and $1 \mathrm{~mm}$ in thickness under the pressure of $200 \mathrm{MPa}$. The specimens were sintered at $1170{ }^{\circ} \mathrm{C}$ for $4 \mathrm{~h}$ in a closed alumina crucible, in which pellets with the same composition as the reference were placed around specimens, in order to prevent a change in composition due to volatilization of $\mathrm{PbO}$. The sintered samples were electroded with silver paste and fired at $600{ }^{\circ} \mathrm{C}$ for $30 \mathrm{~min}$.
2.2 Characterization For the fatigue experiments, the samples were placed between two spherical metal clamps and subjected to the cycling by an application of a bipolar sine wave electric field with the amplitude of $2 \mathrm{kV} \mathrm{mm}^{-1}$ at the frequency of $50 \mathrm{~Hz}$. In order to avoid arcing, the samples were immersed in silicone oil. The different sets of samples were fatigued for $1.8 \times 10^{5}, 3.6 \times 10^{5}, 9 \times 10^{5}$, and $3.8 \times 10^{6}$ cycles.

Several test procedures were performed on the samples fatigued to different cycle number: (i) hysteresis loop measurement, (ii) measurement of the spatial heterogeneity of the piezoelectric constant after partial switching, and (iii) switching charge measurement during the polarization reversal by an application of rectangular electric field pulses.

The polarization hysteresis loop $(P-E)$ was measured using a Sawyer-Tower capacitive voltage divider with a $10 \mu \mathrm{F}$ low-leakage sensing capacitor in a computerized measuring system. A triangular bipolar electric field waveform (amplitude of $2 \mathrm{kV} \mathrm{mm}^{-1}$ at the frequency of $1 \mathrm{~Hz}$ ) was applied using a high voltage amplifier (Trek 609B, Trek, USA) driven by an arbitrary waveform generator (33220A, Agilent, USA). Voltage from the capacitor proportional to the switched charge was buffered by an electrometric operational amplifier and recorded by a multifunctional data acquisition board.

For the study of polarization reversal in rectangular electric field pulses the time dependence of the switching charge was measured by a test system similar to one described elsewhere [13]. To characterize the fatigue heterogeneity, the samples fatigued at different number of cycles were divided into segments with the equal effective electrode area. The switching charge measurements were performed on the whole sample as well as on the separated segments. DC voltage of $2.0 \mathrm{kV}$ was supplied by a high voltage supply buffered by a high voltage capacitor of $0.33 \mu \mathrm{F}$ in order to support the current flowing during polarization reversal. In order to limit the maximum current of the whole circuit a $100 \Omega$ series resistance was used. The voltage proportional to switching charge was measured from $10 \mu \mathrm{F}$ low-leakage sensing capacitor connected in series with the sample. Data were recorded by a digital oscilloscope (DPO 3014, Tektronix, USA). High voltage pulses were generated by a high voltage MOSFET switch (HTS 81, Behlke, Germany) controlled by the waveform generator (33220A, Agilent, USA). Samples after different fatigue cycles were first poled for $200 \mathrm{~s}$ and then switched by the rectangular pulses of opposite polarity with the durations of $1 \mathrm{~ms}, 1 \mathrm{~s}$, and $100 \mathrm{~s}$. After each switching the samples were poled again for $100 \mathrm{~s}$.

To study the spatial heterogeneity of the piezoelectric constant during polarization reversal the piezoelectric measurements were done after partially switching of the samples. All samples were initially poled $\left(2.0 \mathrm{kV} \mathrm{mm}^{-1}, 2 \mathrm{~h}\right.$ at room temperature). Then the rectangular electric field pulses with a duration from 0.001 to $20 \mathrm{~s}$ and the amplitude of $2.0 \mathrm{kV} \mathrm{mm}^{-1}$ were applied in order to partially switch the samples. After each polarization switching and the piezoelectric measurements the samples were back-switched 
to the poled state using a pulse with the opposite polarity and the longer duration than used for the switching pulse. The switching pulses were applied using the high voltage amplifier (Trek 609B, Trek, USA) driven by the arbitrary waveform generator (33220A, Agilent, USA). For the measurements of piezoelectric constant $d_{33}$ a conventional Berlincourt-type (stress-induced charge) quasi-static $d_{33}$ meter with a top spherical probe of $1 \mathrm{~mm}$ radius was used with an applied force of $0.2 \mathrm{~N}$ at a frequency of $100 \mathrm{~Hz}$.

The poling voltage applied to the top electrode was negative (relative to the bottom electrode) and switching voltage was positive. This means that after poling the polarization was directed toward the top electrode and the measured $d_{33}$ value was positive. Otherwise, the polarization reversal during the application of the switching pulse led to the negative value of $d_{33}$.

To measure the heterogeneity of the $d_{33}$ values the measurements were taken on a $5 \times 5$ points square grid over the electrode area after each partial switching. The broad range of the pulse duration allows performing the detailed comparison of switching characteristics in all samples with different fatigue stages.

3 Results and discussion $P-E$ loops measured after different number of cycles show strong fatigue behavior (Fig. 1) with a substantial reduction of the remnant polarization $P_{\mathrm{r}}$ compared to the fresh sample.

The local piezoelectric properties can be obtained by the quasi-static $d_{33}$ measurements during the polarization reversal process. Figure 2 shows an example of the evolution of $d_{33}$ spatial distribution over the sample area during the polarization reversal obtained by the partial switching using different pulse durations (data for $1.8 \times 10^{5}$ cycles is shown). After the poling $\left(0.0 \mathrm{~s}\right.$ in Fig. 2) the piezoelectric constant $d_{33}$ is at its maximum value and nearly uniform across the

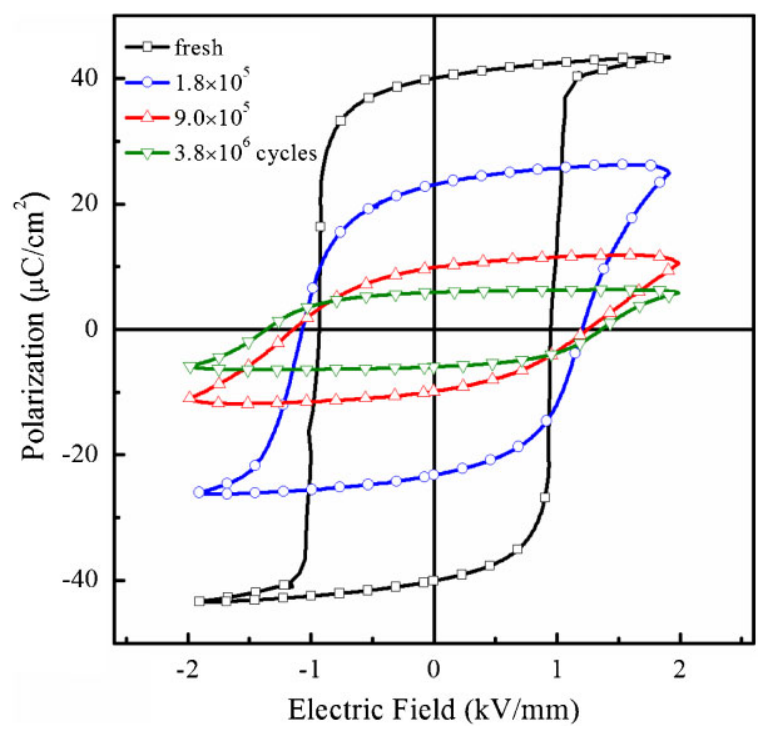

Figure 1 Ferroelectric $(P-E)$ hysteresis loops measured after certain number of switching cycles.

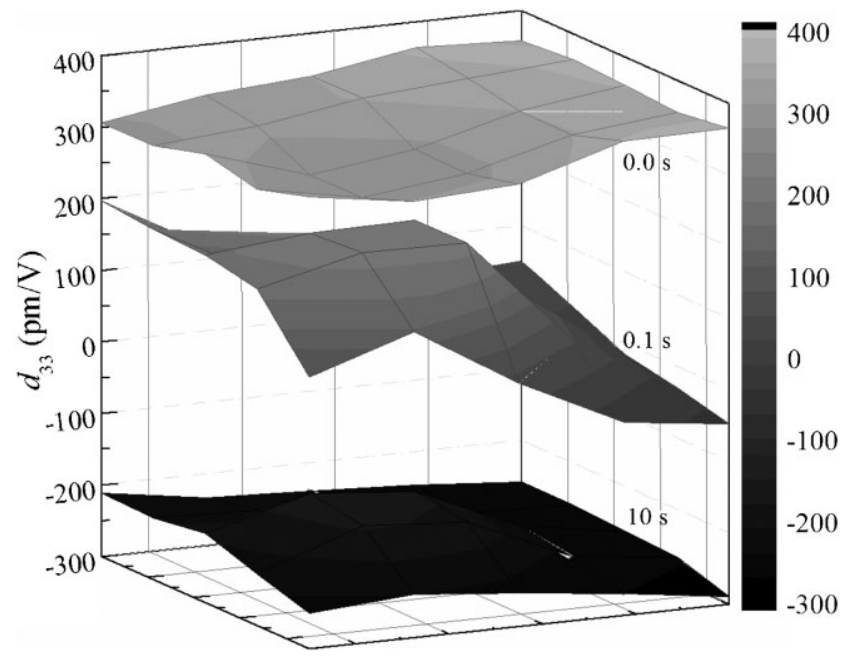

Figure 2 Three-dimensional plot of the spatial distribution of the $d_{33}$ value measured by the quasi-static Berlincourt method over the

sample. During the polarization reversal, the $d_{33}$ spatial distribution becomes essentially non-uniform intermediate surface in Fig. 2, corresponding to pulse duration of $1.0 \mathrm{~s}$ due to the heterogeneous switching in the fatigued sample. It should be noted that the $d_{33}$ value is more or less uniform after the poling and after the complete switching in opposite direction, but becomes strongly non-uniform during the switching process. The change of the $d_{33}$ spatial distribution during the polarization reversal was characterized statistically by the mean value $\langle d\rangle$, standard deviation $\sigma_{\mathrm{d}}$ and range $R_{\mathrm{d}}=\max \left(d_{33}\right)-\min \left(d_{33}\right)$ calculated after each partial switching for the data measured in the different points across the sample. The mean value changes the sign during polarization reversal while the standard deviation and the range increase essentially during the polarization reversal and decrease when the switching is complete (data for $3.6 \times 10^{5}$ cycles are shown in Fig. 3).

The amplitude of $d_{33}$ change during polarization reversal $A_{\mathrm{d}}$ could be calculated by $A_{\mathrm{d}}=d_{33}(0)-d_{33}(T)$, where $T$ is duration of the switching pulse. An average value across the sample $<A_{\mathrm{d}}>$ can be used for the characterization of the fatigue state of the whole sample (Fig. 3) while the maximum and minimum values can give information about the nonuniformity of the properties.

Time dependence of the range $R_{\mathrm{d}}$ during polarization reversal for samples fatigued to different number of cycles are shown in Fig. 4, demonstrating an essential increase of the spatial non-uniformity during the fatigue process. The behavior of $\sigma_{\mathrm{d}}(t)$ for different cycle numbers is similar to the shown behavior of $R_{\mathrm{d}}(t)$. It should be noted, however, that the behavior of $R_{\mathrm{d}}$ at high number of cycles is affected by the overall decrease of the piezoelectric response values of the sample due to polarization fatigue which naturally leads to a decrease of the absolute values of variation.

In order to compare the relative spatial non-uniformity of the samples fatigued to different cycle numbers the range $R_{\mathrm{d}}$ was normalized to the average amplitude of $d_{33}$ change 


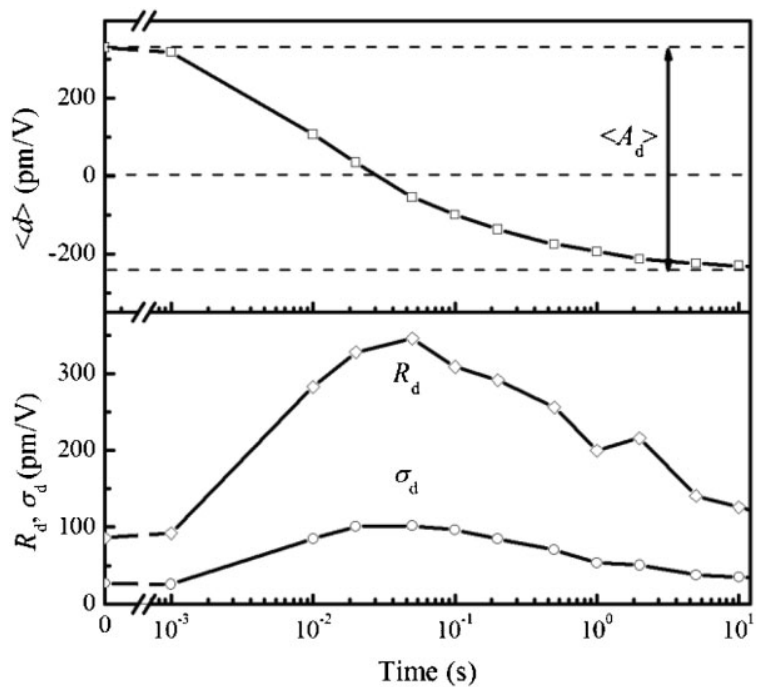

Figure 3 Dependence of the statistical characteristics of the $d_{33}$ spatial distribution for the sample fatigued to $3.6 \times 10^{5}$ cycles on the duration of switching pulse: $\langle d\rangle$ mean value, averaged over the sample area, $\sigma_{\mathrm{d}}$ standard deviation, range $R_{\mathrm{d}}=\max \left(d_{33}\right)-\min$ $\left(d_{33}\right)$ over the sample area. $A_{\mathrm{d}}$ is the amplitude of $d_{33}$ change during polarization reversal.

during polarization reversal $\left(<A_{\mathrm{d}}>\right)$. The maximum and minimum values of $R_{\mathrm{d}} /<A_{\mathrm{d}}>$ during polarization reversal are plotted versus number of cycles in Fig. 5. The maximum value of $R_{\mathrm{d}} /<A_{\mathrm{d}}>$ essentially increases from the beginning of cycling while the minimum one remains practically unchanged. The fast increase of the maximum value with respect to the minimum leads to the conclusion that the heterogeneity is mostly related to the local switching retardation (the increase of the switching time), but not to the loss of the polarization itself.

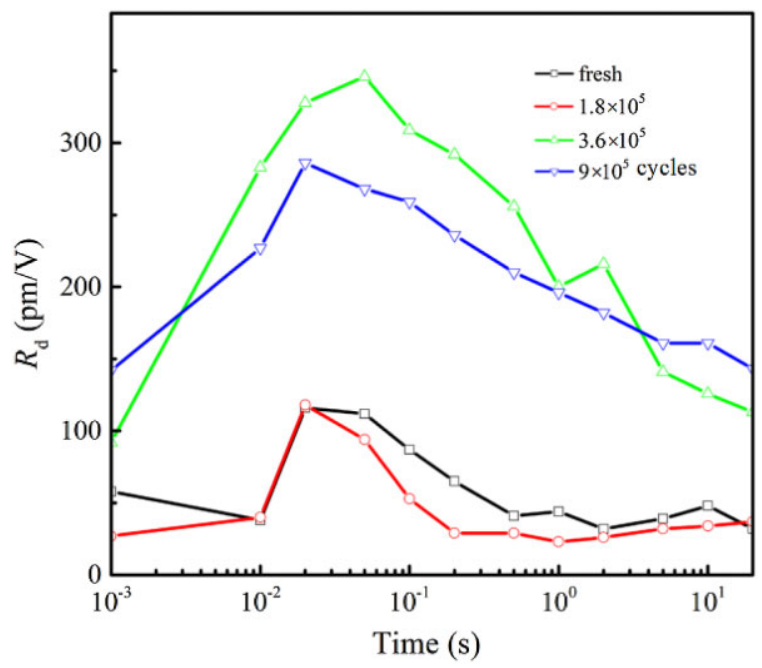

Figure 4 Dependence of the $R_{\mathrm{d}}$ on the switching pulse duration for the samples fatigued to different cycle numbers.

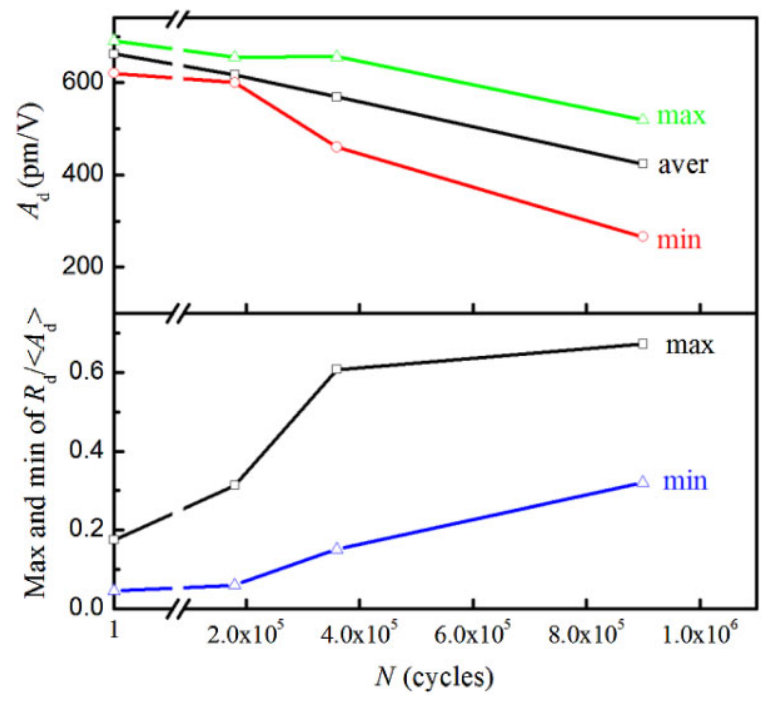

Figure 5 Dependence of maximum, average, minimum values of amplitude $A_{\mathrm{d}}$ and normalized range $R_{\mathrm{d}} /<A_{\mathrm{d}}>$ on the number of cycles.

The fatigue process is also accompanied by the decrease of the averaged and maximum values of $A_{\mathrm{d}}$ (Fig. 5) which correlates to the decrease of $P_{\mathrm{r}}$. It should be noted, that the maximum value of $A_{\mathrm{d}}$ is decreased only by $25 \%,<A_{\mathrm{d}}>$ is decreased by $40 \%$ while the minimum value is dropped more than twice (Fig. 5) showing buildup of significant heterogeneity of the switching characteristics during cycling. Nevertheless, the relative change of $A_{\mathrm{d}}$ even for minimum value is significantly lower than for $P_{\mathrm{r}}$ (4 times for the same number of cycles) which can be attributed to two factors: first of all, the polarization loop measurements from which $P_{\mathrm{r}}$ values are derived are made in the varying field (triangular pulses), while the measurements of the $d_{33}$ during polarization reversal are made in the constant field regime (rectangular pulses) with the value of the DC field equal to the amplitude of the triangular pulses. Thus the higher field applied for a longer time leads to more complete switching in rectangular pulses, especially for strongly fatigued samples. An additional reason for the difference is the relatively low spatial resolution of $d_{33}$ measurements during switching which can lead to missing of some highly fatigued regions of the sample in statistics, especially close to the electrode edge where the mechanical reasons of fatigue like cracks are more probable.

The present measurements of the local piezoelectric constant may, at least qualitatively, demonstrate the fatigue heterogeneity $[8,10]$ in PZT ceramics. It is known that the dependence of piezoelectric constant $d_{33}$ on the polarization $P_{3}$ can be described as follows [14]:

$$
d_{33}(E)=2 Q_{\mathrm{eff}} P_{3}(E) \varepsilon_{33},
$$

where $Q_{\text {eff }}$ is the effective electrostrictive coefficient and $\varepsilon_{33}$ is the dielectric constant. This equation is insufficient to 
describe polycrystal properties, because effectively hysteretic ferroelastic behavior is encountered. The changes of material response due to the application of electric field $E$ and stress as well as fatigue cycling up to cycle numbers $N$ can be included in an analogue dependence [1]:

$$
d_{33}(E, \sigma, N)=2 Q_{\mathrm{eff}} P_{3}(E, \sigma, N) \varepsilon_{33}(E, \sigma, N) .
$$

A generalization of the correlation between the piezoelectric constant $d_{33}$ and the polarization $P_{3}$ is presented for our above discussion. Next, we show that the heterogeneous distribution displayed in Fig. 2 is associated with the fatigue states of the PZT samples.

Time dependence of switching charge density shows an essential increase of switching time with fatigue cycling (Fig. 6) similar to the $d_{33}$ measurements. Moreover, essential sample heterogeneity after the fatigue cycling was revealed. An example is shown in Fig. 7 in which the time dependence of the switching charge measured on different segments of the samples is presented along with a curve measured on the whole sample area (dashed), which represents the average behavior. Even for $1.8 \times 10^{5}$ cycles, pulse duration required to switch half of the polarization differs up to three orders of magnitude depending on the position of the segment in the sample, but the switching is nevertheless complete for each part. This remarkable fact clearly demonstrates the appearance of the heterogeneity of the switching properties during the fatigue cycling. It is worth to note that the most significant changes are noticed in the switching time value while the maximum switching charge (Fig. 6) is decreased only $25 \%$ for $10^{6}$ switching cycles.

The observed essential dispersion of characteristic local switching time is in accordance with a model proposed for the explanation of the switching behavior in PZT thin films taking into account a distribution of the switching time [15].

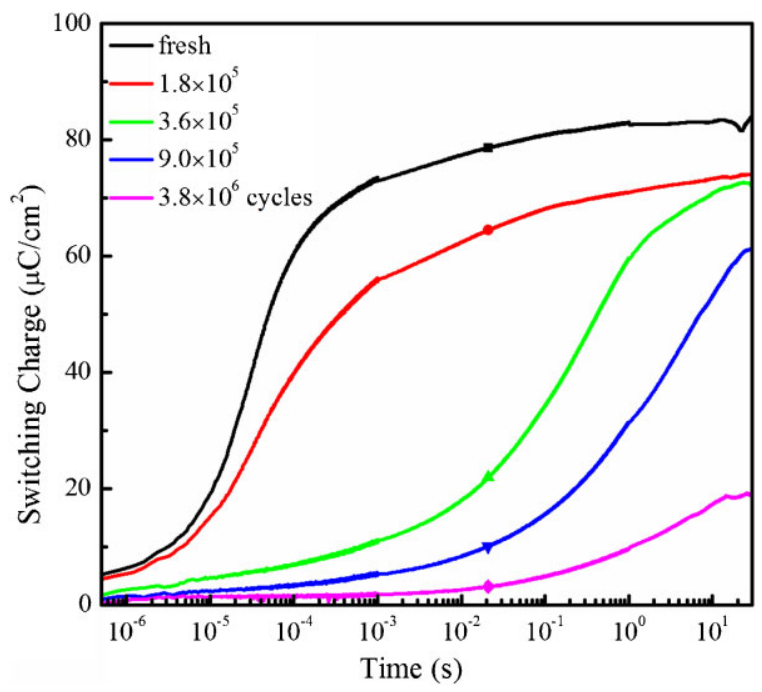

Figure 6 Time dependence of the switching charge density for the samples fatigued to different number of cycles.

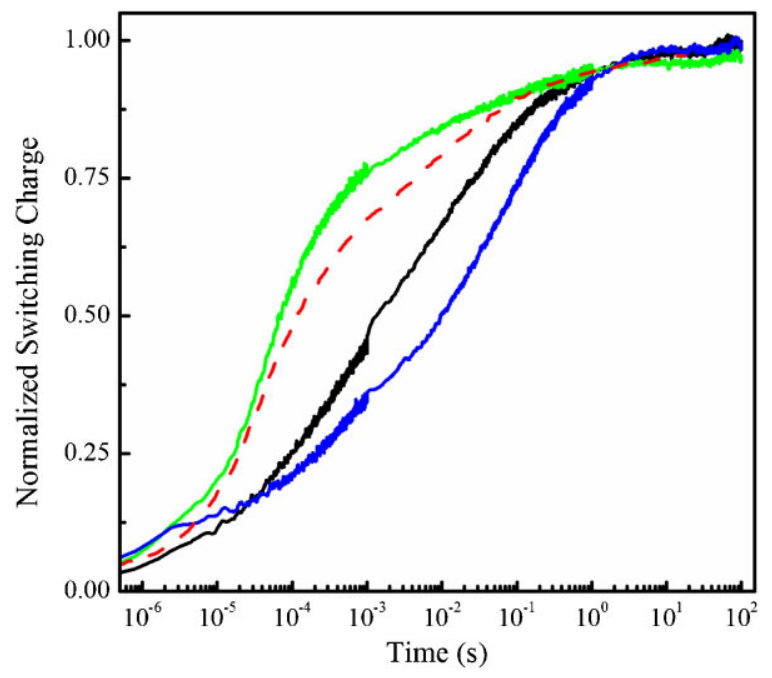

Figure 7 Time dependence of the normalized switching charge density measured in different segments (solid curves) and whole electrode (dashed curve) for the sample fatigued to $1.8 \times 10^{5}$ cycles.

The remarkable fact that the change of $P_{\mathrm{r}}$ differs essentially from the changes of $d_{33}$ amplitude and maximum value of the switching charge leads to the conclusion that the fatigue characterization should be done carefully using several techniques or using different pulse time/frequencies. Moreover, the pulse parameters of a preferable procedure for fatigue testing should be as close as possible to the real device application regime.

It should be noted that polarization and switching charge were measured in slowly varying or constant electric field in DC regime while the piezoelectric response was measured by AC excitation at $100 \mathrm{~Hz}$. Comparison of the results obtained in the different regimes is justified in our case by the fact that we are mostly discussing the heterogeneity of the parameters, but not their direct relations. Moreover, the comparison of $P-E$ and strain loop data with smallsignal $\mathrm{AC} d_{33}$ measurements usually gives reasonable results $[1,8]$.

The changes of the piezoelectric and switching properties can be explained in terms of formation of frozen domains (domains which cannot be switched after certain number of cycles) [16]. According to a kinetic approach to the fatigue phenomenon proposed by Shur et al. [17], the fatigue can be considered as a formation of the frozen domain induced by the buildup of the spatially non-uniform bias field during cycling. Such non-uniform bias field leads to the local variation of the switching time thus resulting in the observed heterogeneity of the switching characteristics in different regions of the sample. The heterogeneity of the local switching times is also the reason of observed tilting of the hysteresis loops $[18,19]$.

In contrast with the switching charge measurements the piezoelectric signal includes not only switchable polarization, but the input of frozen domains as well [20]. The 
measured $d_{33}$ distribution during polarization reversal was used to estimate the role of frozen domains. Local offset of piezoelectric signal $d_{\text {off }}$ was calculated from the time dependence of $d_{33}$ for each part of the sample. It was found that all offsets are of one sign, corresponding to the preferable orientation of the frozen domain in the poling direction. No correlation was found between the offset and the amplitude of $d_{33}$ change for all studied samples. Moreover, the average offset was found to decrease with fatigue cycling from 175 to $100 \mathrm{pm} \mathrm{V}^{-1}$ with relatively small standard deviation (increased from 9 to $20 \mathrm{pm} \mathrm{V}^{-1}$ during cycling). The observed unipolarity of the frozen part of the polarization is believed to be due to poling stage used before the beginning of polarization reversal study. Such behavior suggests that the most part of frozen domains can be indeed switched by the application of external field applied for longer time thus demonstrating their kinetic properties.

It should be pointed out that the measured polarization switching and piezoelectric properties are averaged over the thickness of the sample. Moreover, the transversal resolution is fairly low. Thus the observed heterogeneity has a macroscopic scale, much larger than the possible heterogeneity scale due to arbitrary orientation of the grains or their properties. This fact can be attributed to the influence of space charges on grain boundaries, electrical field inside a grain on its neighbourhoods as well as mechanical clamping. Moreover, formation of macroscopic frozen domains can be attributed to transgrain domain growth mechanism demonstrated previously [21].

4 Conclusions The increase of spatial non-uniformity of the switching properties during the fatigue cycling in lead zirconate titanate ceramics using both the quasi-static $d_{33}$ measurements and the polarization switching measurements was studied. The statistical analysis of spatial variation of the switching properties and its evolution with cycle number provides the evidence that the heterogeneity is mostly related to the non-uniform change of the local characteristic switching time. Moreover, the observed preferable orientation of frozen non-switchable domains in one direction is due to the long-time poling stage used before start of the measurement. Finally, the formation of frozen domains and spatially non-uniform field in the ceramics during fatigue accounts for the spatial heterogeneity of piezoelectric properties.
Acknowledgments This work was supported by National Natural Science Foundation of China (Grant No. 50672042), the Ministry of Sciences and Technology of China through 973-Project under Grant No. 2009CB623306 and Russian Foundation for Basic research (Grant No. 11-02-91174-NSFC-a). IB acknowledge the financial support of young scientists in terms of Ural Federal University development program.

\section{References}

[1] D. C. Lupascu and J. Roedel, Adv. Eng. Mater. 7, 882 (2005).

[2] A. K. Tagantsev, I. Stolichnov, E. L. Colla, and N. Setter, J. Appl. Phys. 90, 1387 (2001).

[3] N. Balke, D. C. Lupascu, T. Blair, and A. Gruverman, J. Appl. Phys. 100, 114117 (2006).

[4] J. J. Lee, C. L. Thio, and S. B. Desu, J. Appl. Phys. 78, 5073 (1995).

[5] W. L. Warren and D. Dimos, J. Appl. Phys. 77, 6695 (1995).

[6] D. Wang and Y. Fotinich, J. Appl. Phys. 83, 5342 (1998).

[7] E. L. Colla, I. Stolichnov, P. E. Bradely, and N. Setter, Appl. Phys. Lett. 82, 1604 (2003).

[8] Y. Zhang, D. C. Lupascu, E. Aulbach, I. Baturin, A. Bell, and J. Roedel, Acta. Mater. 53, 2203 (2005).

[9] J. W. Li, Y. Zhang, H. R. Cai, and X. X. Yi, J. Electroceram. 25, 135 (2010).

[10] Y. Zhang, I. S. Baturin, E. Aulbach, D. C. Lupascu, A. L. Kholkin, V. Y. Shur, and J. Roedel, Appl. Phys. Lett. 86, 012910 (2005).

[11] X. H. Du, J. Zheng, U. Belegundu, and K. Uchino, Appl. Phys. Lett. 72, 2421 (1998).

[12] S. Y. Chu, T. Y. Chen, I. T. Tsai, and W. Water, Sens. Actuators A 113, 198 (2004).

[13] D. C. Lupascu, S. Fedosov, C. Verdier, J. Roedel, and H. von Seggern, J. Appl. Phys. 95, 1386 (2004).

[14] W. Y. Pan, W. Y. Gu, D. J. Taylor, and L. E. Cross, Jpn. J. Appl. Phys. 28, 653 (1989).

[15] A. K. Tagantsev, I. Stolichnov, N. Setter, J. S. Cross, and M. Tsukada, Phys. Rev. B 66, 214109 (2002).

[16] E. L. Colla, S. Hong, D. V. Taylor, A. K. Tagantsev, N. Setter, and K. No, Appl. Phys. Lett. 72, 2763 (1998).

[17] V. Y. Shur, E. L. Rumyantsev, E. V. Nikolaeva, E. I. Shishkin, and I. S. Baturin, J. Appl. Phys. 90, 6312 (2001).

[18] Y. Zhang and I. S. Baturin, Ferroelectrics 398, 1 (2010).

[19] V. Y. Shur, I. S. Baturin, E. I. Shishkin, and M. V. Belousova, Integr. Ferroelectrics 53, 379 (2003).

[20] V. Y. Shur, E. L. Rumyantsev, E. Nikolaeva, E. Shishkin, I. S. Baturin, A. Shur, D. C. Lupascu, M. Ozgul, and C. A. Randall, Proc. SPIE 4699, 40 (2002).

[21] W. Cao and C. A. Randall, J. Phys. Chem. Solids 57, 1499 (1996). 